\title{
Alternative Local Discriminant Bases Using Empirical Expectation and Variance Estimation
}

\author{
Eirik Fossgaard, ${ }^{*}$
}

June 22, 2021

\begin{abstract}
We propose alternative discriminant measures for selecting the best basis among a large collection of orthonormal bases for classification purposes. A generalization of the Local Discriminant Basis Algorithm of Saito and Coifman is constructed. The success of these new methods is evaluated and compared to earlier methods in experiments.
\end{abstract}

\section{Introduction}

This paper is the result of my trying to improve the method applied in Fossgaard (1997) to discriminate between two distinct classes/types of signals by using expansions of the data in wavelet packet/local trigonometric bases. This method was first invented and described by N.Saito and R.Coifman. For a thorough exposition on this theme, I refer to Saito (1994) and Saito, Coifman (1996), a brief summary of the main ideas is given below.

Each signal belonging to a training dataset is decomposed in a time/space -frequency dictionary, that is a decomposition into a large collection of orthonormal bases arranged in a binary tree structure, containing either wavelet-packet basis functions, or local trigonometric basis functions. A measure of energydensity is then computed for each coordinate in the dictionary for each class of signals, originally in Saito (1994) this is taken to be the square of the coordinate summed over all the training signals belonging to a class of signals, and then normalized by the total energy projected onto this coordinate. Then a basis called the "Local Discriminant Basis", LDB for short, is chosen from the dictionary by maximizing a certain discrimination measure, defined by some additive cost-functional, over the dictionaries of energy-densities. The coordinates where the discrimination measure takes on its largest values are called the most important features of the signals. These coordinates are selected from the LDB and used as input for some classifier.

This method is very powerful in many cases, but it also has its weaknesses, a serious one is that the LDB is not able to distinguish two signals both consisting

\footnotetext{
*Eirik Fossgaard is currently a lecturer at Institute of Mathematics and Statistics, University of Troms $\varnothing, 9037$ Troms $\varnothing$, Norway (E-mail: eirikf@math.uit.no). This work was supported by Department of Mathematics, Royal Institute of Technology, 10044 Stockholm, Sweden. The author thanks Professor Jan Olov Strömberg, Department of Mathematics, Royal Institute of Technology, 10044 Stockholm, Sweden, for helpful suggestions.
} 
exclusively of one and the same basis element, only with opposite sign. One way of dealing with this problem is described in Saito, Coifman (1996), where one estimates the probability-density functions, pdf's, of the projections onto the different basis elements in the dictionary, and selects the basis which maximizes some well-chosen functional on these pdf's.

In this paper, I will try to improve on the LDB-method described above, by constructing new dicrimination measures that yield more relevant features. I will also try to improve the performance of the algorithm by using several LDB's in sequence, and by using a classifier specially designed to fully utilize the increased degree of freedom multiple LDB's (MLDB's) give us in selecting features that are most important to our problem.

\section{The original LDB method}

The problem as expressed in Saito (1994) is optimizing a linear map: $d: \mathcal{X} \rightarrow \mathcal{Y}$, where $\cup_{y \in \mathcal{Y}} \mathcal{X}^{(y)}=\mathcal{X} \subset \mathbf{R}^{n}$ is the input signal space, $\mathcal{Y}=\{1,2, \ldots, N\}$ is the output class space, a set of class labels, and $\mathcal{X}^{(y)}$ is the subspace of class $y$ signals. To optimize the map $d$, one considers maps of the form

$$
d=c \circ \mathcal{F}_{K} \circ \boldsymbol{\Psi}_{n \times n},
$$

where the feature extractor $\boldsymbol{\Psi}_{n \times n} \in O(n)$ is an orthogonal $n \times n$ matrix which extracts the $n$ most relevant coordinates from from a binary-tree dictionary of wavelet packet bases or local trigonometric bases, $\mathcal{F}_{K}$ is a feature selector which selects the $K<n$ most important coordinates from the $n$ most relevant coordinates, and $c$ is a classifier. The problem then is to choose $c, \mathcal{F}_{K}$ and $\boldsymbol{\Psi}_{n \times n}$ such that the rate of misclassification of the map $d$ is minimized on the set $\mathcal{X}$. In Saito (1994), $\boldsymbol{\Psi}_{n \times n}$ is taken to be

$$
\boldsymbol{\Psi}_{n \times n}=\arg \max _{B_{k} \in \mathcal{D}_{i} \in \mathcal{L}} \lambda\left(B_{k}\right),
$$

where $\mathcal{L}=\cup_{i} \mathcal{D}_{i}$ is the library of all dictionaries at our disposal corresponding to the different wavelet or local trigonometric basis functions under consideration, the $B_{k}$ are all bases in $\mathcal{D}_{i}$, and $\lambda$ is a measure of performance of the basis $B_{k}$ in the classification problem, such a measure is called a discrimination measure. The search for this $\boldsymbol{\Psi}_{n \times n}$ is fast by the best-basis-algorithm of Wickerhauser and Coifman if the measure $\lambda$ satisfies an additivity property, (Saito 1994). In Saito (1994) the discrimination measure $\lambda$ is defined as

$$
\lambda\left(B_{k}\right)=\sum_{\mathbf{w}_{m} \in B_{k}} \gamma\left(\Gamma^{(1)}\left(\mathbf{w}_{m}\right), \ldots, \Gamma^{(N)}\left(\mathbf{w}_{m}\right)\right),
$$

where the time-frequency energy-map $\Gamma^{(y)}$ is defined by

$$
\begin{aligned}
& \Gamma^{(y)}\left(\mathbf{w}_{m}\right)=\frac{\sum_{j=1}^{J_{y}}\left(\mathbf{w}_{m} \cdot \mathbf{x}_{j}^{(y)}\right)^{2}}{\sum_{j=1}^{J_{y}}\left\|\mathbf{x}_{j}^{(y)}\right\|^{2}}, \\
& \mathbf{x}_{j}^{(y)} \in \mathcal{X}^{(y)}, 1 \leq y \leq N, J_{y}=\left|\mathcal{X}^{(y)}\right|,
\end{aligned}
$$

and $\gamma$ can be some form of $l^{p}$ - distance, Hellinger-distance or relative entropy. 
The signals $\mathbf{x}^{(y)} \in \mathcal{X}^{(y)}, 1 \leq y \leq N$ are fed into each dictionary as given by (11), the best basis picked out by the best basis algorithm, and then the best $K$ coordinates are selected from this basis, ordinarily by selecting the coordinates where $\lambda$ takes on its $K$ greatest values. The corresponding $K$ best basis elements are then used to construct a classifier by doing a "Linear Discriminant Analysis" (LDA) or a "Classification and Regression Trees" (CART)-analysis, or some other statistical classification technique, on the coordinates of the signals in these $K$ best basis elements.

\section{A generalized LDB method}

\subsection{New Discrimination Measures}

Using the notation from the previous section, for each basis vector $\mathbf{w}_{m}$ in some basis $B_{k}$, let $Z_{y, m}$ be random variable on the space $\mathcal{X}^{(y)}$ of input signals of class $y$ defined by

$$
Z_{y, m}: \mathbf{x} \in \mathcal{X}^{(y)} \rightarrow[-1,1], Z_{y, m}(\mathbf{x})=\mathbf{w}_{m} \cdot \mathbf{x} .
$$

In Saito, Coifman (1996) one estimates the empirical pdf $p$ of $Z_{y, m}$. These estimates are then used to find the most discriminating basis. But getting good estimates of the pdf's is hard and computationally demanding. We will take a different approach and work on the a priori assumption that $p$ is the uniform distribution. For each fixed $\mathbf{w}_{m} \in B_{k}$, we can then compute the empirical expectation $E\left[Z_{y, m}\right]$ of the basis coordinate $\mathbf{w}_{m} \cdot \mathbf{x}$ for class $y$ signals as

$$
\begin{aligned}
E\left[Z_{y, m}\right] & =\sum_{\mathcal{X}^{(y)}} p\left(Z_{y, m} \mid Y=y\right) Z_{y, m} \\
& =\sum_{\mathbf{x} \in \mathcal{X}^{(y)}} \frac{1}{\left|\mathcal{X}^{(y)}\right|}\left(\mathbf{w}_{m} \cdot \mathbf{x}\right) .
\end{aligned}
$$

If $\|\mathbf{x}\|_{2}=1, \forall \mathbf{x} \in \mathcal{X}$, then in this probabilistic setting, (田) is equivalent to $\Gamma^{(y)}\left(\mathbf{w}_{m}\right)=E\left[Z_{y, m}^{2}\right]$. We will first consider two-class problems: $\mathcal{Y}=\{1,2\}$, and deal with $n$-class problems later. Choosing $\gamma=\ell^{2}$-distance squared, (3) becomes

$$
\lambda\left(B_{k}\right)=\sum_{m: \mathbf{w}_{m} \in B_{k}}\left(E\left[Z_{1, m}^{2}\right]-E\left[Z_{2, m}^{2}\right]\right)^{2} .
$$

We see that with this $\lambda$, the best basis given by (2) is the basis maximizing the sum of the euclidean distances between the expected values of all the basis coordinates for the two classes. Now, we observe that the measure of performance (7) of the basis $B_{k}$ does not consider how the data is distributed around the expected values. For example, if:

$$
\begin{aligned}
& I_{1, m}=\left[E\left[Z_{1, m}^{2}\right]-\sqrt{\operatorname{Var}\left[Z_{1, m}^{2}\right]}, E\left[Z_{1, m}^{2}\right]+\sqrt{\operatorname{Var}\left[Z_{1, m}^{2}\right]}\right] \\
& I_{2, m}=\left[E\left[Z_{2, m}^{2}\right]-\sqrt{\operatorname{Var}\left[Z_{2, m}^{2}\right]}, E\left[Z_{2, m}^{2}\right]+\sqrt{\operatorname{Var}\left[Z_{2, m}^{2}\right]}\right]
\end{aligned}
$$

where $\operatorname{Var}\left[Z_{y, m}^{2}\right]$ is empirical variance of $Z_{y, m}^{2}$, then it may well happen that $I_{1, m} \cap I_{2, m} \neq \emptyset$, even if $\mathbf{w}_{m} \in \arg \max _{B_{k} \in \mathcal{D}_{i} \in \mathcal{L}} \lambda\left(B_{k}\right)$. 
Ideally, we want a basis $B$ where the overlap $\mathcal{O}(B)$ given by

$$
\mathcal{O}(B)=\sum_{m: \mathbf{w}_{m} \in B}\left|I_{1, m} \cap I_{2, m}\right|
$$

is as small as possible. That is a basis which simultanously is discriminating between classes and has the opposite property inside classes. This motivates the following definition of a new discrimination measure $\lambda^{\prime}$ by

$$
\lambda^{\prime}\left(B_{k}\right)=\sum_{m: \mathbf{w}_{m} \in B_{k}}\left[\frac{E\left[Z_{1, m}^{2}\right]-E\left[Z_{2, m}^{2}\right]}{\left(\operatorname{Var}\left[Z_{1, m}^{2}\right]+\operatorname{Var}\left[Z_{2, m}^{2}\right]\right)^{1 / 2}}\right]^{2} .
$$

Note how the performance measure in (8) defers from the measure in (7). We see that the numerator in (8) measures the separability of datapoints between the classes 1,2 , and the denominator measures the dispersion of the datapoints inside each of the classes 1,2 . Neither of the measures $\lambda^{\prime}, \lambda$ captures differences between classes in sign in the basis coordinates. To improve on this fact, we define the measure $\lambda^{\prime \prime}$ by

$$
\begin{aligned}
\lambda^{\prime \prime}\left(B_{k}\right)= & \sum_{m: \mathbf{w}_{m} \in B_{k}}\left[E\left[\left(Z_{1, m}-Z_{2, m}\right)^{2}\right]^{1 / 2} /\right. \\
& \left(E\left[\left(Z_{1, m}(\mathbf{x})-Z_{1, m}\left(\mathbf{x}^{\prime}\right)\right)^{2}\right]^{1 / 2}+\right. \\
& \left.\left.E\left[\left(Z_{2, m}\left(\mathbf{x}^{\prime \prime}\right)-Z_{2, m}\left(\mathbf{x}^{\prime \prime \prime}\right)\right)^{2}\right]^{1 / 2}\right)\right], \\
& \mathbf{x} \neq \mathbf{x}^{\prime} \in \mathcal{X}^{(1)}, \mathbf{x}^{\prime \prime} \neq \mathbf{x}^{\prime \prime \prime} \in \mathcal{X}^{(2)} .
\end{aligned}
$$

We see that the numerator in (9) measures the separability of signed datapoints between the classes 1,2 , and the denominator measures the dispersion of signed datapoints inside these classes.

\subsection{Construction of an Oracle Classifier Using Multiple LDB's}

The construction is due to the following observation: Having chosen a best basis $\boldsymbol{\Psi}_{n \times n}^{t}$, where $\boldsymbol{\Psi}_{n \times n}=\arg \max _{B_{k} \in \mathcal{D}_{i} \in \mathcal{L}} \zeta\left(B_{k}\right)$, and $\zeta$ is some discrimination measure, there are subsets $\mathcal{S}_{j}$ of the set $\mathcal{X}$ of input signals on which $\boldsymbol{\Psi}_{n \times n}^{t}$ works better than other subsets. That is, the signals in disjoint sets $\mathcal{S}_{j}$ have significant differences in how they distribute their energy among the different elements in the basis $\boldsymbol{\Psi}_{n \times n}^{t}$. More precisely: Let $\mathcal{W}_{K}$ be the feature space of dimension $K<n$ spanned by the $K$ most important elements in the best basis $\boldsymbol{\Psi}_{n \times n}^{t}$, sorted in decreasing order of importance, and $P_{\mathcal{W}_{K}}$ be the orthogonal projection onto $\mathcal{W}_{K}$.

Now, consider the sets $A^{(k)}$ and $B^{(k)}$ of points in $k$-dimensional euclidean space given by: $A^{(k)}=\left\{P_{\mathcal{W}_{k}} \mathbf{x}_{j}\right\}_{\mathbf{x}_{j} \in \mathcal{X}^{(1)}} \subset[-1,1]^{k}, B^{(k)}=\left\{P_{\mathcal{W}_{k}} \mathbf{x}_{j}\right\}_{\mathbf{x}_{j} \in \mathcal{X}^{(2)}}$ $\subset[-1,1]^{k}$. It is clear by the definition of $\mathcal{W}_{k}$, that the two point-clouds $A^{(k)}$ and $B^{(k)}$ should be concentrated in more or less disjoint regions in $[-1,1]^{k}$ if the two classes are separable by our method, that is we should observe clustering when plotting the points of $A^{(k)}$ and $B^{(k)}$ in $[-1,1]^{k}$ and labeling each point after its class.

We sort out clusters by the following recursive algorithm. 
Algorithm 3.1 The Dyadic Cluster Search Algorithm (DCSA). Given appropriately chosen numbers $n \geq K \geq 1,1>\delta>0,1>\eta \geq 0,1>\mu \geq \nu>0$.

Step 0: Choose a performance measure $\lambda$ as in (1), (\$) or in (\$), or some other favourite measure. Set $\left.\beta=\lceil\nu|\mathcal{X}|\rceil, \gamma_{A}=\left\lceil\eta \mid \mathcal{X}^{(1)}\right\rceil\right\rceil, \gamma_{B}=\left\lceil\eta\left|\mathcal{X}^{(2)}\right|\right\rceil, \quad I=$ $[-1,1]$.

Step 1: Select the feature spaces $\mathcal{W}_{K}$ by the formula (目) and truncate to the $K<n$ most important basis elements. Compute the sets $A^{(K)}, B^{(K)}$ as defined above. Set $\Delta=0.0, k=1, C^{(k)}=I^{k}, C_{\text {next }}^{(k)}=I^{k}$, FoundCluster $=0$.

Step 2: Set $A=A^{(k)}, B=B^{(k)}, C=C^{(k)}, C_{\text {next }}=C_{\text {next }}^{(k)}$. If $|A| \leq$ $\gamma_{A}$ and $|B| \leq \gamma_{B}$, terminate the algorithm. Else, compute $\alpha=\lceil\mu(|A|+|B|)\rceil$, $N_{A}(C)=|A \cap C|, N_{B}(C)=|B \cap C|$.

Step 3: If $N_{A}+N_{B} \geq \max (\alpha, \beta)$, compute the error rate $\epsilon=\min \left(N_{A}, N_{B}\right) /\left(N_{A}+\right.$ $\left.N_{B}\right)$ and proceed to the next step. Else, if $C_{n e x t} \neq C$, set $C=C_{\text {next }}$ and jump to Step 2. Else, if $C_{\text {next }}=C$, and FoundCluster $=1$, jump to Step 1. Else, if $C_{\text {next }}=C$, FoundCluster $=0$, if $k<K$, set $k=k+1$ and jump to Step 2 . Else, if $C_{\text {next }}=C$, FoundCluster $=0, k=K$, set $k=1, \Delta=\Delta+\delta$ and jump to Step 2.

Step 4: If $\epsilon \leq \Delta$, store the location of the cube $C$ together with the numbers $N_{A}, N_{B}$ and identification of the $k$ basis elements defining the space $\mathcal{W}_{k}$. Then, for each index $i \in\{1,2, \ldots, K\}$, set $A^{(i)}=A^{(i)}-\left(P_{\mathcal{W}_{i}} A^{(K)}\right) \cap C, B^{(i)}=B^{(i)}-$ $\left(P_{\mathcal{W}_{i}} B^{(K)}\right) \cap C$ and for each $\mathbf{x}_{j_{i}} \in \mathcal{X}^{(i)}$, if $P_{\mathcal{W}_{k}} \mathbf{x}_{j_{i}} \in C, \mathcal{X}^{(i)}=\mathcal{X}^{(i)}-\mathbf{x}_{j_{i}}, i=$ 1,2. Set FoundCluster $=1, \Delta=0.0, k=1$, and jump to Step 2. Else, divide $C$ into $2^{k}$ subcubes $C_{1}, \ldots, C_{2^{k}}$ by splitting each of the sidelengths of $C$ into two sides of equal length, and for each index $i=1,2, \ldots, 2^{k}$, jump to Step 2 with $C=C_{i}, C_{\text {next }}=C_{i+1}, 1 \leq i<2^{k}, C_{\text {next }}=C, i=2^{k}$.

Less precisely: This algorithm carries out a classification on the signals in the input signal space $\mathcal{X}=\mathcal{X}^{(1)} \cup \mathcal{X}^{(2)}$ by dividing the set $\mathcal{X}$ into disjoint subsets $\mathcal{S}_{j}$ and performing a classification on each of these subsets represented in a basis $\boldsymbol{\Psi}_{j}^{t}$. Each $\mathcal{S}_{j}$ consists exclusively of the signals $\mathbf{x}_{i} \in \mathcal{X}$ on which the most discriminating basis $\boldsymbol{\Psi}_{j}^{t}$ selected by (2) performs best. Having computed a best basis $\boldsymbol{\Psi}_{1}^{t}$, the set $\mathcal{S}_{1}$ is selected first, the signals in $\mathcal{S}_{1}$ are assigned class names and then $\mathcal{S}_{1}$ is deleted from the set $\mathcal{X}$. Then a new best basis $\Psi_{2}^{t}$ for the new $\mathcal{X}$ is computed by the formula (2), the set $\mathcal{S}_{2}$ is selected, and so on. The algorithm terminates when the set $\mathcal{X}$ has become sparse. Thus, we see that by adapting the parameters we can prevent the algorithm from trying to classify the part of the training dataset which it finds most difficult to classify, and so we gain a smaller overall training-error-rate. But this adjusting of parameters has to be done carefully, so that the algorithm does not fail to catch important features of the signals. The algorithm selects the subsets $\mathcal{S}_{j}$ using as few features as possible, starting with only the most important feature element (= the most discriminating basis element in the best basis). Then, 
given some upper limit on the rate of error allowed in the clusters, if no clean clustering is observed in the feature space of this single feature element, the algorithm adds information by taking into consideration also the second best feature element and looks for clustering in the feature space spanned by the two best feature elements and so on. If no clean clustering is observed using all $K$ best feature elements, the upper error limit is increased and the feature space of the one most important feature element is again searched for clusters, and so on. Using as few features as possible reduces the risk of overtraining of the algorithm, that is the algorithm selecting features that are too adapted to the specific set $\mathcal{X}$ of training data. On the other hand, we see that this algorithm is flexible in its selection of relevant features in that it constructs a sequence of feature extractors $\left\{\boldsymbol{\Psi}_{j}^{t}\right\}$ where each $\boldsymbol{\Psi}_{j}^{t}$ is specially adapted to some part $\mathcal{S}_{j}$ of the dataset $\mathcal{X}$. The output of the algorithm is a sequence $\mathcal{C}=\left\{C_{i}\right\}_{i=1}^{L}$ of dyadic hypercubes $C_{i} \subset[-1,1]^{K}$ of possibly different dimensions $k_{i}, 1 \leq k_{i} \leq K, 1 \leq i \leq L$, where to each cube $C_{i}$ corresponds a specific feature space $\mathcal{W}_{k_{i}}$ as defined above, and a class name $y_{C_{i}}$ which equals the name of the majority class of the set $\left(\left\{P_{\mathcal{W}_{k_{i}}} \mathbf{x}_{j}\right\}_{\mathbf{x}_{j} \in \mathcal{X}}\right) \cap C_{i}=\left\{P_{\mathcal{W}_{k_{i}}} \mathbf{x}_{j}\right\}_{\mathbf{x}_{j} \in \mathcal{S}_{i}}$ of datapoints in $\mathcal{W}_{k_{i}}$ that $C_{i}$ contains. We will call $\mathcal{C}$ a simple two-class oracle classifier, or simply oracle, for the two-class problem $d: \mathcal{X}^{(1)} \cup \mathcal{X}^{(2)} \rightarrow \mathcal{Y}=\{1,2\}$.

\subsection{On Using and Choosing Oracle Classifiers}

Given a two-class problem $d: \mathcal{X}^{(1)} \cup \mathcal{X}^{(2)} \rightarrow \mathcal{Y}=\{1,2\}$, we compute $\mathcal{C}=$ $\left\{C_{j}\right\}_{j=1}^{L}$ by the DCSA. Then, given a sample $\mathbf{x} \in \mathcal{T}$, where $\mathcal{T}$ is a test dataset, we assign $\mathbf{x}$ to a class by the following procedure: We check if: $P_{\mathcal{W}_{k_{j}}} \mathbf{x} \in C_{j}$, starting with index $j=1$ and continuing until we get a positive answer for some index $j^{\prime} \leq L$. We then assign a weighted class $y_{C_{j^{\prime}}}$-vote to $\mathrm{x}$ by computing the product of $1-\epsilon_{j^{\prime}}$, where $\epsilon_{j^{\prime}}$ is the error rate of $C_{j^{\prime}}$, and its statistical frequency $\left(N_{A}\left(C_{j^{\prime}}\right)+N_{B}\left(C_{j^{\prime}}\right)\right) /|\mathcal{X}|$. If $P_{\mathcal{W}_{k_{j}}} \mathbf{x} \notin C_{j}, \forall C_{j} \in \mathcal{C}$, we consider the class of $\mathbf{x}$ undetermined.

Different choices of discrimination measure or different settings of the parameters in the DCSA result in different classifiers. For a two-class problem, we can construct several classifiers by using different performance measures/parameters, and let the weighted majority vote of the classifiers decide whether a sample $\mathbf{x} \in \mathcal{T}$ is of class 1 or class 2 . For a $n$-class problem, $n>2$, we will apply the method of splitting the $n$-class problem into $n$ two-class problems: $d: \mathcal{X} \rightarrow\{i, 0\}, 1 \leq i \leq n$, as proposed in Saito, Coifman (1996), by splitting the training data set into two sets of class $i$ and not $i$. One then constructs oracles for each two-class problem. To classify an unknown sample $\mathbf{x} \in \mathcal{T}$, we compute weighted class votes as explained above for the set of oracles and assign $\mathbf{x}$ to the majority vote class.

\section{Experimental Results}

In some of the calls to the DCSA in the experiments described below we allowed the algorithm to select a best basis only once, we call this method a LDB-method (Local Discriminant Basis-method). In the cases were we allowed the algorithm to select multiple different best bases in sequence, we call the method a MLDBmethod (Multiple Local Discriminant Basis-method). In the cases where we 
organized the classifiers resulting from different calls (calls with different discrimination measures) to the DSCA into a classifier by taking the majority vote over these classifiers, we call the method a superposition LDB or MLDB-method, denoted SLDB or SMLDB-method, respectively. In all the three examples below we generated 10 independent realizations of both the training dataset and the test dataset. The results shown in Table 1, Table 2, Table 3 are the mean over the 10 simulations corresponding to the 10 independent realizations of the datasets.

\subsection{Example 1}

We consider a two class waveform classification problem as presented in Fossgaard (1997). We generated sets of 100 training signals and 1000 test signals of length 1024 for each class by the formula

$$
\begin{aligned}
& Q_{n}(R, \theta, t)= \\
& C(R, t) \sum_{j=1}^{n} A_{n}(j) e^{i k\left(\frac{r_{j}^{2}}{2 R}-r_{j} \cos \left(\theta-\theta_{j}\right)\right)},
\end{aligned}
$$

where we have:

$$
C(R, t)=\frac{e^{-i k(c t-R)}}{R} \text { is considered constant }=1
$$

for simplicity.

$R=10^{4}$.

$k=100$.

$A_{n}(j)=\frac{1}{n}$.

$r_{j}$ is random variable uniformly distributed on $[1,10]$.

$\theta_{j}$ is random variable uniformly distributed on

$$
\left[2 \pi \frac{j}{n}, 2 \pi \frac{j}{n}+\frac{\pi}{4}\right] .
$$

For each $n$-tiple of realizations $\left\{r_{j}, \theta_{j}\right\}_{j=1}^{n}$ of the pair of random variables $r_{j}, \theta_{j}$, we generate a discrete signal $S_{n}(\theta)$ by uniformly sampling the real part of $Q_{n}(R, \theta, t) 1024$ times in the variable $\theta$ with sampling density $2 \pi / 16 \cdot k=$ $2 \pi / 1600$. We generated data sets by extracting realizations of $S_{n} /\left\|S_{n}\right\|_{2}$ smoothly from a fixed sampling interval. In this problem we used $n=3, n=4$ in (10) to define two classes of signals and the coiflet with filterlength 18 as dictionary. All calls to the DCSA in this experiment were made with $K=5, \delta=0.01, \eta=$ $0.05, \mu=0.10, \nu=0.05$. The results are shown in Table 1 .

\subsection{Example 2}

This example is identical to Example 1 except that we used $n=4, n=5$ in $(10)$ to define the two signal classes. We used the coiflet with filterlength 18 as dictionary. All calls to the DCSA in this experiment were made with $K=5, \delta=0.01, \eta=0.05, \mu=0.10, \nu=0.05$. The results are shown in Table 2. 


\begin{tabular}{lcccccccc}
\hline \hline Method & \multicolumn{3}{c}{ Classification rate (\%) } & \multicolumn{3}{c}{ Error rate (\%) } \\
& \multicolumn{2}{c}{ Training data } & \multicolumn{2}{c}{ Test data } & \multicolumn{2}{c}{ Training data } & \multicolumn{2}{c}{ Test data } \\
& Total & $\sigma$ & Total & $\sigma$ & Total & $\sigma$ & Total & $\sigma$ \\
\hline LDB1 & 99.7 & 0.7 & 99.5 & 0.9 & 19.9 & 3.0 & 29.8 & 2.8 \\
\hline MLDB1 & 98.5 & 1.4 & 98.4 & 1.4 & 8.6 & 1.1 & 23.5 & 2.7 \\
\hline LDB2 & 97.6 & 2.0 & 96.3 & 3.6 & 16.0 & 4.5 & 23.5 & 4.4 \\
\hline MLDB2 & 95.2 & 1.9 & 93.8 & 2.3 & 12.9 & 3.1 & 23.7 & 3.4 \\
\hline LDB3 & 98.9 & 1.7 & 98.8 & 2.1 & 16.6 & 4.6 & 24.6 & 4.9 \\
\hline MLDB3 & 98.4 & 1.1 & 99.5 & 0.6 & 13.7 & 3.1 & 24.4 & 1.9 \\
\hline SLDB & 100 & 0.0 & 100 & 0.0 & 14.7 & 3.9 & 22.2 & 1.5 \\
\hline SMLDB & 100 & 0.0 & 100 & 0.0 & 9.1 & 3.3 & $\mathbf{2 0 . 4}$ & 2.0 \\
\hline \hline
\end{tabular}

Table 1: The average classification rates and the corresponding error rates over 10 simulations from Example 1. LDB1 is the LDB selected by the measure $\lambda^{\prime}$. MLDB1 is the MLDB selected by the measure $\lambda^{\prime}$. LDB2 is the LDB selected by the measure $\lambda^{\prime \prime}$. MLDB2 is the MLDB selected by the measure $\lambda^{\prime \prime}$. LDB3 is the LDB selected by the measure $\lambda$. MLDB3 is the MLDB selected by the measure $\lambda$. SLDB is the superposition of methods LDB1, LDB2, LDB3. SMLDB is the superposition of the methods MLDB1, MLDB2, MLDB3. $\sigma$ is the square root of the sample variance.

\subsection{Example 3}

We consider a three class waveform classification problem as presented in Saito (1994). We generated sets of 100 training signals and 1000 test signals of length 32 for each class by first extracting signal samples by the formulas

$$
\begin{aligned}
& f_{1}(i)=u h_{1}(i)+(1-u) h_{2}(i)+\epsilon(i) \text { for Class } 1 \\
& f_{2}(i)=u h_{1}(i)+(1-u) h_{3}(i)+\epsilon(i) \text { for Class } 2 \\
& f_{3}(i)=u h_{2}(i)+(1-u) h_{3}(i)+\epsilon(i) \text { for Class } 3
\end{aligned}
$$

where $i=1, \ldots, 32, h_{1}(i)=\max (6-|i-7|, 0), h_{2}(i)=h_{1}(i-8), h_{3}(i)=h_{1}(i-4)$, $u$ is a uniform random variable on the interval $(0,1)$, and $\epsilon(i)$ are the standard normal variates. We then normalized the signals in the energy norm by setting $f_{1}(i)=f_{1}(i) /\left\|f_{1}\right\|_{2}, f_{2}(i)=f_{2}(i) /\left\|f_{2}\right\|_{2}, f_{3}(i)=f_{3}(i) /\left\|f_{3}\right\|_{2}, i=1, \ldots, 32$. We used the coiflet with filterlength 6 as a dictionary for this problem. All calls to the DCSA in this experiment were made with $K=5, \delta=0.01, \eta=0.05, \mu=$ $0.20, \nu=0.05$. The results are shown in Table 3 .

\section{Comments}

\subsection{Comments to Example 1}

In this example we achieved the best result by the superposition method using multiple LDB's, denoted SMLDB. We see that the generalized methods MLDB1, MLDB2, MLDB3 are almost indistinguishable in this example, we conclude that our new measures $\lambda^{\prime}, \lambda^{\prime \prime}$ hardly yield a significantly better classification than the original measure $\lambda$, the positive effect is in any case small. Furtermore, for the measure $\lambda^{\prime}$ we do get better results by the generalized method, whereas for 


\begin{tabular}{lcccccccc}
\hline \hline Method & \multicolumn{3}{c}{ Classification rate (\%) } & \multicolumn{3}{c}{ Error rate (\%) } \\
& \multicolumn{2}{c}{ Training data } & \multicolumn{2}{c}{ Test data } & \multicolumn{2}{c}{ Training data } & \multicolumn{2}{c}{ Test data } \\
& Total & $\sigma$ & Total & $\sigma$ & Total & $\sigma$ & Total & $\sigma$ \\
\hline LDB1 & 99.3 & 1.4 & 98.7 & 2.3 & 11.9 & 3.5 & 19.9 & 5.4 \\
\hline MLDB1 & 98.0 & 1.4 & 97.3 & 2.3 & 6.4 & 2.9 & 20.5 & 4.3 \\
\hline LDB2 & 96.9 & 2.6 & 96.9 & 2.5 & 10.5 & 2.7 & $\mathbf{1 7 . 5}$ & 3.2 \\
\hline MLDB2 & 96.0 & 2.2 & 94.1 & 3.6 & 9.5 & 1.9 & 19.0 & 2.6 \\
\hline LDB3 & 99.1 & 1.7 & 99.6 & 0.9 & 24.2 & 5.6 & 32.6 & 7.9 \\
\hline MLDB3 & 98.5 & 1.4 & 99.5 & 0.5 & 21.6 & 3.6 & 35.8 & 4.2 \\
\hline SLDB & 100 & 0.0 & 100 & 0.0 & 15.0 & 4.9 & 21.8 & 4.5 \\
\hline SMLDB & 100 & 0.0 & 100 & 0.0 & 8.4 & 3.7 & 20.1 & 3.2 \\
\hline \hline
\end{tabular}

Table 2: The average classification rates and the corresponding error rates over 10 simulations from Example 2. LDB1 is the LDB selected by the measure $\lambda^{\prime}$. MLDB1 is the MLDB selected by the measure $\lambda^{\prime}$. LDB2 is the LDB selected by the measure $\lambda^{\prime \prime}$. MLDB2 is the MLDB selected by the measure $\lambda^{\prime \prime}$. LDB3 is the LDB selected by the measure $\lambda$. MLDB3 is the MLDB selected by the measure $\lambda$. SLDB is the superposition of methods LDB1, LDB2, LDB3. SMLDB is the superposition of the methods MLDB1, MLDB2, MLDB3. $\sigma$ is the square root of the sample variance.

the measures $\lambda^{\prime \prime}$ and $\lambda$ the positive effect of generalizing is more doubtful. But all in all, it seems we are a little better off with either measure $\lambda^{\prime}, \lambda^{\prime \prime}$ than the original $\lambda$.

\subsection{Comments to Example 2}

In this example we achieved the best result with the method LDB2. We see that both discrimination measures $\lambda^{\prime}, \lambda^{\prime \prime}$ clearly outperform the original measure $\lambda$ in this problem. As in the previous example, the measures $\lambda^{\prime}$ and $\lambda^{\prime \prime}$ yield about the same results with MLDB-methods. When not taking superpositions of several classifiers, the generalised MLDB-method does not yield any improvements in results on test data, rather it seems that this method adapts too much to training data in this example. Furthermore, due to the poor performance of the measure $\lambda$ in this example, we get worse results with superposition methods in this example than when using the best single classifier. But we could expect to further lower the best error rate on test data by combining classifiers from the measures $\lambda^{\prime}, \lambda^{\prime \prime}$ only.

\subsection{Comments to Example 3}

In this example we achieved the best result by the method SMLDB, and we see that superposition methods are clearly favourable in this case. However, it seems to make little difference which measure we are using when not taking superpositions of several classifiers. We remark that both the measures $\lambda^{\prime}, \lambda^{\prime \prime}$ select the standard basis as the most discriminating basis in the first steps in the DCSA, whereas $\lambda$ does not choose this basis in any step. 


\begin{tabular}{lcccccccc}
\hline \hline Method & \multicolumn{3}{c}{ Classification rate (\%) } & \multicolumn{4}{c}{ Error rate (\%) } \\
& \multicolumn{2}{c}{ Training data } & \multicolumn{2}{c}{ Test data } & \multicolumn{2}{c}{ Training data } & \multicolumn{2}{c}{ Test data } \\
& Total & $\sigma$ & Total & $\sigma$ & Total & $\sigma$ & Total & $\sigma$ \\
\hline LDB1 & 100 & 0.0 & 100 & 0.0 & 23.7 & 1.9 & 28.2 & 0.8 \\
\hline MLDB1 & 100 & 0.0 & 100 & 0.0 & 22.9 & 2.1 & 28.5 & 2.7 \\
\hline LDB2 & 100 & 0.0 & 100 & 0.0 & 23.6 & 2.4 & 27.9 & 1.9 \\
\hline MLDB2 & 100 & 0.0 & 100 & 0.0 & 20.7 & 2.7 & 27.7 & 1.5 \\
\hline LDB3 & 100 & 0.0 & 100 & 0.0 & 25.0 & 2.6 & 29.1 & 1.8 \\
\hline MLDB3 & 100 & 0.0 & 100 & 0.0 & 23.0 & 2.6 & 26.2 & 2.6 \\
\hline SLDB & 100 & 0.0 & 100 & 0.0 & 18.7 & 1.9 & 22.7 & 0.9 \\
\hline SMLDB & 100 & 0.0 & 100 & 0.0 & 15.7 & 1.9 & $\mathbf{2 0 . 5}$ & 1.0 \\
\hline \hline
\end{tabular}

Table 3: The average classification rates and the corresponding error rates over 10 simulations from Example 3. LDB1 is the LDB selected by the measure $\lambda^{\prime}$. MLDB1 is the MLDB selected by the measure $\lambda^{\prime}$. LDB2 is the LDB selected by the measure $\lambda^{\prime \prime}$. MLDB2 is the MLDB selected by the measure $\lambda^{\prime \prime}$. LDB3 is the LDB selected by the measure $\lambda$. MLDB3 is the MLDB selected by the measure $\lambda$. SLDB is the result from a superposition of the methods LDB1, LDB2, LDB3. SMLDB is the result from a superposition of the methods MLDB1, MLDB2, MLDB3. $\sigma$ is the square root of the sample variance.

\subsection{Conclusion}

We have shown that estimating expectations and variances directly from the expansion coefficients of the datasets in the binary-tree structured dictionary of bases may lead to better results than when using the energy-density dictionaries of bases. Also, we have shown that comparing/combining different discrimination measures in classification problems may lead to significant improvements in the success of the classification methods.

\section{A Applied software and hardware}

All algorithms and transforms used in the numerical experiments, except some of the random number generators described below, were implemented in the computer language $\mathrm{C}++$ and compiled with the GNU project $\mathrm{C}++$ compiler on a HP K260 machine with a PA 8000 processor.

\section{A.1 Random number generators}

In the examples we used the Fortran NAG-routines G05DAF, G05FAF for generating random numbers with uniform distribution, and G05FDF for generating random numbers with standard normal distribution.

\section{References}

[1] Saito, N.(1994), "Local Feature Extraction and Its Applications Using a Library of Bases", dissertation, Yale University. 
[2] Saito, N.,and Coifman, R.(1996), "Improved Local Discriminant Bases Using Empirical Probability Density Estimation", Proceedings of Statistical Computing.

[3] Fossgaard, E.(1997), "Fast Computational Algorithms for the Discrete Wavelet Transform and Applications of Localized Orthonormal Bases in Signal Classification", md-thesis, University of Troms $\varnothing$. 